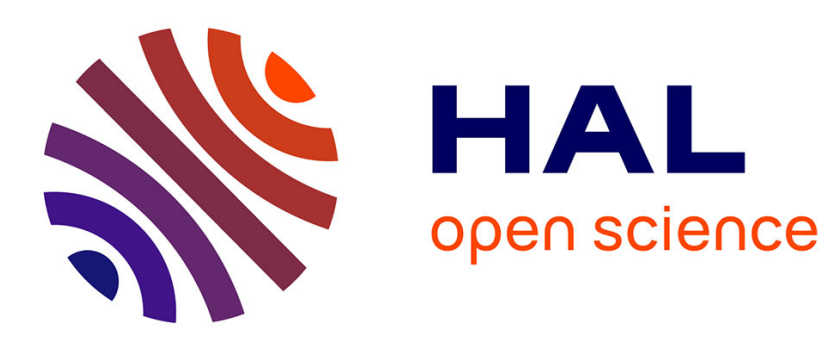

\title{
A regime switching model to evaluate bonds in a quadratic term structure of interest rates
}

Stéphane Goutte, Raphaël Homayoun, Thomas Porcher

\section{To cite this version:}

Stéphane Goutte, Raphaël Homayoun, Thomas Porcher. A regime switching model to evaluate bonds in a quadratic term structure of interest rates. 2014. hal-01090846

\section{HAL Id: hal-01090846 https://hal.science/hal-01090846}

Preprint submitted on 9 Dec 2014

HAL is a multi-disciplinary open access archive for the deposit and dissemination of scientific research documents, whether they are published or not. The documents may come from teaching and research institutions in France or abroad, or from public or private research centers.
L'archive ouverte pluridisciplinaire HAL, est destinée au dépôt et à la diffusion de documents scientifiques de niveau recherche, publiés ou non, émanant des établissements d'enseignement et de recherche français ou étrangers, des laboratoires publics ou privés. 


\title{
A regime switching model to evaluate bonds in a quadratic term structure of interest rates.
}

\author{
Stéphane GOUTTE *†, Raphaël Homayoun BOROUMAND ${ }^{\ddagger}$ and Thomas PORCHER ${ }^{\S}$
}

February 18, 2014

\begin{abstract}
In this article, we consider a discrete time economy in which we assume that the short term interest rate follows a quadratic term structure in a regime switching asset process. The possible non-linear structure and the fact that the interest rate can have different economic or financial trends justify Regime Switching Quadratic Term Structure Model (RS-QTSM). Indeed, this regime switching process depends on the values of a Markov chain with a time dependent transition probability matrix which can capture the different states (regimes) of the economy. We prove that under this model, the conditional zero coupon bond price admits a quadratic term structure. Moreover, the stochastic coefficients which appear in this decomposition satisfy an explicit system of coupled stochastic backward recursions.
\end{abstract}

Keywords: Quadratic term structure model; Regime switching; Zero coupon bond; Markov chain.

\footnotetext{
${ }^{*}$ Universit Paris 8 (LED), 2 rue de la Libert, 93526 Saint-Denis Cedex, France

${ }^{\dagger}$ Affiliated Professor, ESG Management School, 25 rue Saint-Ambroise 75011 Paris France.

${ }^{\ddagger}$ Associate Professor, Department of Applied Economics, ESG Management School, 25 rue Saint-Ambroise 75011 Paris France.

$\S$ Associate Professor, Department of Applied Economics, ESG Management School, 25 rue Saint-Ambroise 75011 Paris France.
} 
MSC classification: 60J10 91B25 91G30.

JEL classification: G10, G12, G15.

\section{Introduction}

Modeling the term structure of interest rates has long been an important topic in economics and finance. Most of the papers on modelling of the interest rate term structure are related to the family of the Affine Term Structure Models (ATSM). These models consider a linear relation between the log price of a zero coupon bond and its states factors. These models have first been studied by Vasicek (1977) in [16] and Cox, Ingersoll and Ross (1985) in [4]. They have been further developed by Duffie and Kan (1996) in [6] and Dai and Singleton (2000) in [5]. A first extension of this class of models was to use a regime switching model. Thus, Elliott et al. (2011) in [8] considered a discrete-time, Markov, regime-switching, affine term structure model for valuing bonds and other interest rate securities. Recently, Goutte and Ngoupeyou (2013) in [11] obtained explicit formulas to price a defaultable bond under this class of regime switching models. The proposed model incorporates the impact of structural changes in economic conditions on interest rate dynamics and can capture different economic (financial) levels or trends of the economy. A second extension was to not only consider a linear model but to model the term structure of interest rates with Quadratic Term Structure Models (QTSM). This family, first introduced by Beaglehole and Tammey (1991) in [2] are applied to price contingent claims (Lieppold and $\mathrm{Wu}(2002)$ in [14]) and to the credit risk pricing (Chen, Filipovic and Poor (2004) in [3]). In this article, we propose to use both the previous extension and a regime switching discrete-time version of quadratic term structure models (RS-QTSM).

The economic benefits of such extensions are the following. Firstly, if we look at a historical path of a log price of a zero coupon bond and its states factors, we clearly see that the assumption of a linear structure is too restrictive. It is then natural to consider a quadratic structure 
instead of a linear one to fit better this relation. Secondly, it is obvious that the dynamic of the short term interest is impacted by exogenous factor of the economy such as economic state or credit rating. A way to model these impacts is to use regime-switching models where the parameters of the short term rate are not constant but can change their values depending on the state of the economy. In a recession state, the parameters are different than in a standard economic one.

An important application of term structure models is the valuation of interest rate instruments, such as zero coupon bonds. We will demonstrate that under the regime switching quadratic term structure modeling, the conditional zero coupon bond price of a regime switching asset admits a quadratic decomposition. Moreover, we find that the stochastic coefficients which appear in this decomposition satisfy an explicit system of coupled stochastic backward recursions.

Our article is organized as follows. In section 1, the model is presented and defined. In section 2, the conditional zero coupon bond price is evaluated and we give the corresponding system of coupled stochastic backward recursions.

\section{The model}

We consider a discrete time economy with finite time horizon and time index set $\mathcal{T}:=\{k \mid k=$ $0,1,2, \ldots, T\}$, where $T$ is a positive integer such that $T<\infty$. Let $(\omega, \mathcal{F}, P)$ be a filtered probability space where $P$ is a risk neutral probability. 


\subsection{Markov chain}

Following Elliott et al. in [7], let $\left(X_{k}\right)_{k \in \mathcal{T}}$ be a discrete time Markov chain on finite state space $\mathcal{S}:=\left\{e_{1}, e_{2}, \ldots, e_{N}\right\}$, where $e_{i}$ has unity in the $i^{t h}$ position and zero elsewhere. Thus $\mathcal{S}$ is the set of canonical unit column vectors of $\mathbb{R}^{N}$. In an economic point of view, $X_{k}$ can be viewed as an observable exogenous quantity which can reflect the evolution of the state of the economy. We assume that the time dependent transition probability matrix $Q_{k}:=\left(q_{i j k}\right)_{i, j=1, \ldots, N}$ of $X$ under $P$ is defined by

$$
q_{i j k}=P\left(X_{k+1}=j \mid X_{k}=i\right) .
$$

It also satisfies $q_{i j k} \geq 0$, for all $i \neq j \in \mathcal{S}$ and $\sum_{j=1}^{N} q_{i j k}=1$ for all $i \in \mathcal{S}$. Let $\mathbb{F}^{X}=\left(\mathcal{F}_{k}^{X}\right)_{k \in \mathcal{T}}:=$ $\sigma\left(X_{k}, k \in \mathcal{T}\right)$ which is the $P$ augmented filtration generated by the history of the Markov chain $X$ and $\mathcal{F}_{k}^{X}$ is the $P$-augmented $\sigma$-field generated by the history of $X$ up to and including time $k$. Moreover, following again Elliott et al. in [7] , the semi-martingale decomposition for the Markov chain $X$ is given by

$$
X_{k+1}=Q_{k} X_{k}+M_{k+1}^{X}, \quad k \in\{0,1,2, \ldots, T-1\},
$$

where $\left(M_{k}^{X}\right)$ is an $\mathbb{R}^{N}$-valued martingale increment process (i.e. $\mathbb{E}\left[M_{k+1}^{X} \mid \mathcal{F}_{k}^{X}\right]=0$ ).

\subsection{Asset}

Let $\left(S_{k}\right)_{k \in \mathcal{T}}$ denotes the state asset process and we denote by $\mathbb{F}^{S}=\left(\mathcal{F}_{k}^{S}\right)_{k \in \mathcal{T}}$ the $P$-augmented filtration generated by the process $S$. Finally, we denote by $\mathcal{G}_{k}:=\mathbb{F}_{k}^{S} \vee \mathbb{F}_{k}^{X}$ the global enlarged filtration for all $k \in \mathcal{T}$. Let $\langle.,$.$\rangle denote the inner product in \mathbb{R}^{N}$. Then, for every $k \in$ $\{1,2, \ldots, T\}$, we define the following regime dependent parameters $\kappa_{k}:=\kappa\left(k, X_{k}\right)=\left\langle\kappa, X_{k}\right\rangle$ $\mu_{k}:=\mu\left(k, X_{k}\right)=\left\langle\mu, X_{k}\right\rangle$ and $\sigma_{k}:=\sigma\left(k, X_{k}\right)=\left\langle\sigma, X_{k}\right\rangle$ where $\kappa:=\left(\kappa_{1}, \kappa_{2}, \ldots, \kappa_{N}\right), \mu:=$ 
$\left(\mu_{1}, \mu_{2}, \ldots, \mu_{N}\right)$ and $\sigma:=\left(\sigma_{1}, \sigma_{2}, \ldots, \sigma_{N}\right)$ are $1 \times N$ real-valued vectors. Moreover, we assume that $\sigma_{i}>0$, for all $i \in\{1,2, \ldots, N\}$. Finally, $\varepsilon:=\left(\varepsilon_{k}\right)_{k \in\{1,2, \ldots, T\}}$ are a sequence of independent and identically distributed random variables with law $\mathcal{N}(0,1)$. We assume that $\varepsilon$ and the Markov chain $X$ are independent. Under the risk neutral probability measure $P$ the dynamic of the asset $S$ is governed by the following discrete time, Markov switching model

$$
S_{k+1}=\kappa_{k}+\mu_{k} S_{k}+\sigma_{k} \varepsilon_{k+1}, \quad k=\{0,1, \ldots, T-1\} .
$$

\subsection{Short term interest rate}

Let $\left(r_{k}\right)_{k \in \mathcal{T}}$ denote the process of short term interest rate. We assume that the dynamic of $r_{k}$ is regime dependent and is following a quadratic term structure of the asset process $S_{k}$ which is given by

$$
r_{k}:=r\left(k, X_{k}\right)=a_{0, k}+a_{1, k} S_{k}+a_{2, k} S_{k}^{2}, \quad k \in \mathcal{T}
$$

with $r_{k}:=r\left(k, X_{k}\right)=\left\langle r, X_{k}\right\rangle, r:=\left(r_{1}, r_{2}, \ldots, r_{N}\right), a_{0, k}:=a_{0}\left(k, X_{k}\right)=\left\langle a_{0}, X_{k}\right\rangle, a_{1, k}:=$ $a_{1}\left(k, X_{k}\right)=\left\langle a_{1}, X_{k}\right\rangle$ and $a_{2, k}:=a_{2}\left(k, X_{k}\right)=\left\langle a_{2}, X_{k}\right\rangle$ where $a_{0}, a_{1}$ and $a_{2}$ are real vectors of size $1 \times N$.

Remark 1.1. The Markov chain X can be seen as an economic impact factor. An economic interpretation of this is that the Markov chain can represent a credit rating of a firm A. Indeed, assume that (1.1) models the spread of a firm A, then the Markov chain can be the credit rating of this firm given by an exogenous rating company as Standard and Poors. Therefore it is natural to consider that the dynamic of the spread of the firm A depends on the value of this notation X (for more detail, see Goutte and Ngoupeyou [11] or Goutte [10]). 


\subsection{Zero-coupon Bond price}

Let $P(k, T)$ be the price at time $k \in \mathcal{T}$ of a zero-coupon bond with maturity $T$. Since we are under the risk neutral probability, we have that

$$
P(k, T)=\mathbb{E}\left[\exp \left(-\sum_{t=k}^{T-1} r_{t}\right) \mid \mathcal{G}_{k}\right], \quad k \in \mathcal{T},
$$

with $P(T, T)=1$ and $P(T-1, T)=\exp \left(-r_{T-1}\right)$.

\section{Regime switching quadratic structure formulas}

\subsection{Full history case}

Let us first assume that we know the full history of the Markov chain $X$. We denote by $\tilde{\mathcal{G}}_{k}:=$ $\mathcal{F}_{T}^{X} \vee \mathcal{F}_{k}^{S}, k \in \mathcal{T}$ this enlarged information set. Then we denote by $\tilde{P}(k, T)$ the conditional zero coupon bond price at time $k$ with maturity $T$ given the enlarged filtration $\tilde{\mathcal{G}}_{k}$. We obtain that

$$
\tilde{P}(k, T)=\mathbb{E}\left[\exp \left(-\sum_{t=k}^{T-1} r_{t}\right) \mid \tilde{\mathcal{G}}_{k}\right], \quad k \in \mathcal{T}
$$

with $\tilde{P}(T, T)=1$ and $\tilde{P}(T-1, T)=\exp \left(-r_{T-1}\right)$.

Theorem 2.1. The conditional bon price $\tilde{P}(k, T)$ has an exponential quadratic term structure given for all $k \in \mathcal{T}$ by

$$
\tilde{P}(k, T)=\exp \left\{c_{1, k}+c_{2, k} S_{k}+c_{3, k} S_{k}^{2}\right\}
$$

where the stochastic coefficients $\left(c_{1, k}\right)_{k \in \mathcal{T}},\left(c_{2, k}\right)_{k \in \mathcal{T}}$ and $\left(c_{3, k}\right)_{k \in \mathcal{T}}$ satisfy the system of coupled stochas- 
tic backward recursions given for all $n \in\{1, \ldots, T-1\}$ by

$$
\begin{aligned}
c_{1, n-1}:= & -a_{0, n-1}+c_{1, n}+c_{2, n} \kappa_{n-1}+c_{3, n} \kappa_{n-1}^{2}+\log \left(\left(1-2 c_{3, n} \sigma_{n-1}^{2}\right)^{-1 / 2}\right) \\
& +\frac{c_{2, n}^{2} \sigma_{n-1}^{2}+4 \kappa_{n-1}^{2} \sigma_{n-1}^{2}}{2\left(1-2 c_{3, n} \sigma_{n-1}^{2}\right)}+\frac{2 c_{2, n} \sigma_{n-1}^{2} \kappa_{n-1}}{\left(1-2 c_{3, n} \sigma_{n-1}^{2}\right)}, \\
c_{2, n-1}:= & -a_{1, n-1}+c_{2, n} \mu_{n-1}+c_{3, n} \kappa_{n-1} \mu_{n-1}+\frac{4 \kappa_{n-1} \mu_{n-1} \sigma_{n-1}^{2}+2 c_{2, n} \sigma_{n-1}^{2} \mu_{n-1}}{\left(1-2 c_{3, n} \sigma_{n-1}^{2}\right)}, \\
c_{3, n-1}:= & -a_{2, n-1}+c_{3, n} \mu_{n-1}^{2}+\frac{2 \mu_{n-1}^{2} \sigma_{n-1}^{2}}{\left(1-2 c_{3, n} \sigma_{n-1}^{2}\right)} .
\end{aligned}
$$

with terminal conditions $c_{1, T}=c_{2, T}=c_{3, T}=0$

Proof. We prove this result by backward induction. Thus since $\tilde{P}(T, T)=1$, the exponential quadratic term structure 2.9 is true for $k=T$. Assume now, that the result holds for $k=n$, we would like to prove that this result also holds for $k=n-1$. By the Definition (2.4) and iterated conditional expectation, we obtain

$$
\begin{aligned}
\tilde{P}(n-1, T) & =\mathbb{E}\left[\exp \left(-\sum_{t=n-1}^{T-1} r_{t} \mid \tilde{\mathcal{G}}_{n-1}\right]=\mathbb{E}\left[\mathbb{E}\left[\exp \left(-\sum_{t=n-1}^{T-1} r_{t}\right) \mid \tilde{\mathcal{G}}_{n}\right] \mid \tilde{\mathcal{G}}_{n-1}\right]\right. \\
& =\mathbb{E}\left[\exp \left(-r_{n-1}\right) \mathbb{E}\left[\exp \left(-\sum_{t=n}^{T-1} r_{t}\right) \mid \tilde{\mathcal{G}}_{n}\right] \mid \tilde{\mathcal{G}}_{n-1}\right] \\
& =\exp \left(-r_{n-1}\right) \mathbb{E}\left[\mathbb{E}\left[\exp \left(-\sum_{t=n}^{T-1} r_{t}\right) \mid \tilde{\mathcal{G}}_{n}\right] \mid \tilde{\mathcal{G}}_{n-1}\right]
\end{aligned}
$$

we can use the assumption that the exponential quadratic term structure 2.9 holds for $k=n$. 
We get using (1.1) and (1.2)

$$
\begin{aligned}
& \tilde{P}(n-1, T)= \exp \left(-r_{n-1}\right) \mathbb{E}\left[\exp \left(c_{1, n}+c_{2, n} S_{n}+S_{n} c_{3, n} S_{n}^{2}\right) \mid \tilde{\mathcal{G}}_{n-1}\right] \\
&= \exp \left\{-a_{0, n-1}-a_{1, n-1} S_{n-1}-a_{2, n-1} S_{n-1}^{2}\right\} \times \\
& \mathbb{E}\left[\exp \left\{c_{1, n}+c_{2, n}\left(\kappa_{n-1}+\mu_{n-1} S_{n-1}+\sigma_{n-1} \varepsilon_{n}\right)+c_{3, n}\left(\kappa_{n-1}+\mu_{n-1} S_{n-1}+\sigma_{n-1} \varepsilon_{n}\right)^{2}\right\} \mid \tilde{\mathcal{G}}_{n-1}\right], \\
&= \exp \left\{-a_{0, n-1}-a_{1, n-1} S_{n-1}-a_{2, n-1} S_{n-1}^{2}\right\} \times \\
& \mathbb{E}\left[\operatorname { e x p } \left\{c_{1, n}+c_{2, n}\left(\kappa_{n-1}+\mu_{n-1} S_{n-1}\right)+c_{2, n} \sigma_{n-1} \varepsilon_{n}+c_{3, n}\left(\kappa_{n-1}+\mu_{n-1} S_{n-1}\right)^{2}\right.\right. \\
&\left.\left.\quad+c_{3, n} \sigma_{n-1}^{2} \varepsilon_{n}^{2}+2\left(\kappa_{n-1}+\mu_{n-1} S_{n-1}\right) \sigma_{n-1} \varepsilon_{n}\right\} \mid \tilde{\mathcal{G}}_{n-1}\right] \\
&= \exp \left\{-a_{0, n-1}-a_{1, n-1} S_{n-1}-a_{2, n-1} S_{n-1}^{2}+c_{1, n}+c_{2, n}\left(\kappa_{n-1}+\mu_{n-1} S_{n-1}\right)+c_{3, n}\left(\kappa_{n-1}+\mu_{n-1} S_{n-1}\right)^{2}\right\} \times \\
& \mathbb{E}\left[\exp \left\{c_{2, n} \sigma_{n-1} \varepsilon_{n}+c_{3, n} \sigma_{n-1}^{2} \varepsilon_{n}^{2}+2\left(\kappa_{n-1}+\mu_{n-1} S_{n-1}\right) \sigma_{n-1} \varepsilon_{n}\right\} \mid \tilde{\mathcal{G}}_{n-1}\right] \\
&=\exp \left\{-a_{0, n-1}-a_{1, n-1} S_{n-1}-a_{2, n-1} S_{n-1}^{2}+c_{1, n}+c_{2, n}\left(\kappa_{n-1}+\mu_{n-1} S_{n-1}\right)+c_{3, n}\left(\kappa_{n-1}+\mu_{n-1} S_{n-1}\right)^{2}\right\} \times \\
& \mathbb{E}\left[\exp \left\{f_{n} \varepsilon_{n}+g_{n} \varepsilon_{n}^{2}\right\} \mid \tilde{\mathcal{G}}_{n-1}\right]
\end{aligned}
$$

with $f_{n}:=c_{2, n} \sigma_{n-1}+2\left(\kappa_{n-1}+\mu_{n-1} S_{n-1}\right) \sigma_{n-1}$ and $g_{n}:=c_{3, n} \sigma_{n-1}^{2}$.

Since $\varepsilon:=\left(\varepsilon_{k}\right)_{k \in\{1,2, \ldots, T\}}$ are a sequence of independent and identically distributed random variables with law $\mathcal{N}(0,1)$, we have

$$
\begin{aligned}
\mathbb{E}\left[\exp \left(f_{n} \varepsilon_{n}+g_{n} \varepsilon_{n}^{2}\right) \mid \tilde{\mathcal{G}}_{n-1}\right] & =\int_{\mathbb{R}} \exp \left(f_{n} \varepsilon_{n}+g_{n} \varepsilon_{n}^{2}\right) \frac{1}{(2 \pi)^{1 / 2}} \exp \left(-\frac{1}{2} \varepsilon_{n}^{2}\right) d \varepsilon_{n} \\
& =\frac{1}{(2 \pi)^{1 / 2}} \int_{\mathbb{R}} \exp \left(f_{n} \varepsilon_{n}+g_{n} \varepsilon_{n}^{2}-\frac{1}{2} \varepsilon_{n}^{2}\right) d \varepsilon_{n}
\end{aligned}
$$

Moreover, we have

$$
f_{n} \varepsilon_{n}+g_{n} \varepsilon_{n}^{2}-\frac{1}{2} \varepsilon_{n}^{2}=-\frac{1}{2}\left[\left(\left(1-2 g_{n}\right)^{1 / 2} \varepsilon_{n}-\left(1-2 g_{n}\right)^{-1 / 2} f_{n}\right)^{2}-\left(1-2 g_{n}\right)^{-1} f_{n}^{2}\right] \text {. }
$$


Then, denoting by $\delta_{n}:=\left(1-2 g_{n}\right)^{-1 / 2}$, we obtain

$$
f_{n} \varepsilon_{n}+g_{n} \varepsilon_{n}^{2}-\frac{1}{2} \varepsilon_{n}^{2}=-\frac{1}{2}\left[\left(\delta_{n}^{-1} \varepsilon_{n}-\delta_{n} f_{n}\right)^{2}-\delta_{n}^{2} f_{n}^{2}\right]
$$

Replacing this formula into 2.6) gives

$$
\begin{aligned}
\mathbb{E}\left[\exp \left(f_{n} \varepsilon_{n}+g_{n} \varepsilon_{n}^{2}\right) \mid \tilde{\mathcal{G}}_{n-1}\right] & =\frac{1}{(2 \pi)^{1 / 2}} \int_{\mathbb{R}} \exp \left(-\frac{1}{2}\left[\left(\delta_{n}^{-1} \varepsilon_{n}-\delta_{n} f_{n}\right)^{2}-\delta_{n}^{2} f_{n}^{2}\right]\right) d \varepsilon_{n} \\
& =\frac{1}{(2 \pi)^{1 / 2}} \int_{\mathbb{R}} \exp \left(-\frac{1}{2}\left(\delta_{n}^{-1} \varepsilon_{n}-\delta_{n} f_{n}\right)^{2}+\frac{1}{2} \delta_{n}^{2} f_{n}^{2}\right) d \varepsilon_{n} \\
& =\frac{1}{(2 \pi)^{1 / 2}} \exp \left(\frac{1}{2} \delta_{n}^{2} f_{n}^{2}\right) \int_{\mathbb{R}} \exp \left(-\frac{\left(\delta_{n}^{-1} \varepsilon_{n}-\delta_{n} f_{n}\right)^{2}}{2}\right) d \varepsilon_{n} \\
& =\delta_{n} \exp \left(\frac{\delta_{n}^{2} f_{n}^{2}}{2}\right) \frac{1}{\delta_{n}(2 \pi)^{1 / 2}} \int_{\mathbb{R}} \exp \left(-\frac{1}{2}\left(\frac{\varepsilon_{n}-\delta_{n}^{2} f_{n}}{\delta_{n}}\right)^{2}\right) d \varepsilon_{n} \\
& =\delta_{n} \exp \left(\frac{\delta_{n}^{2} f_{n}^{2}}{2}\right) .
\end{aligned}
$$

We obtain that

$$
\begin{aligned}
\tilde{P}(n-1, T)= & \exp \left\{-a_{0, n-1}-a_{1, n-1} S_{n-1}-a_{2, n-1} S_{n-1}^{2}+c_{1, n}+c_{2, n}\left(\kappa_{n-1}+\mu_{n-1} S_{n-1}\right)\right\} \\
& \times \exp \left\{c_{3, n}\left(\kappa_{n-1}+\mu_{n-1} S_{n-1}\right)^{2}\right\} \delta_{n} \exp \left\{\frac{\delta_{n}^{2} f_{n}^{2}}{2}\right\}, \\
= & \exp \left\{-a_{0, n-1}-a_{1, n-1} S_{n-1}-a_{2, n-1} S_{n-1}^{2}+c_{1, n}+c_{2, n}\left(\kappa_{n-1}+\mu_{n-1} S_{n-1}\right)\right\} \\
& \times \exp \left\{c_{3, n}\left(\kappa_{n-1}+\mu_{n-1} S_{n-1}\right)^{2}\right\}\left(1-2 g_{n}\right)^{-1 / 2} \exp \left\{\frac{\left(1-2 g_{n}\right)^{-1} f_{n}^{2}}{2}\right\}, \\
= & \exp \left\{-a_{0, n-1}-a_{1, n-1} S_{n-1}-a_{2, n-1} S_{n-1}^{2}+c_{1, n}+c_{2, n}\left(\kappa_{n-1}+\mu_{n-1} S_{n-1}\right)\right\} \\
& \times \exp \left\{c_{3, n}\left(\kappa_{n-1}+\mu_{n-1} S_{n-1}\right)^{2}\right\}\left(1-2 c_{3, n} \sigma_{n-1}^{2}\right)^{-1 / 2} \\
& \times \exp \left\{\frac{\left(1-2 c_{3, n} \sigma_{n-1}^{2}\right)^{-1}\left(c_{2, n} \sigma_{n-1}+2\left(\kappa_{n-1}+\mu_{n-1} S_{n-1}\right) \sigma_{n-1}\right)^{2}}{2}\right\},
\end{aligned}
$$




$$
\begin{aligned}
= & \exp \left\{-a_{0, n-1}-a_{1, n-1} S_{n-1}-a_{2, n-1} S_{n-1}^{2}+c_{1, n}+c_{2, n} \kappa_{n-1}+c_{2, n} \mu_{n-1} S_{n-1}\right\} \\
& \times \exp \left\{c_{3, n} \kappa_{n-1}^{2}+c_{3, n} \mu_{n-1}^{2} S_{n-1}^{2}+c_{3, n} \kappa_{n-1} \mu_{n-1} S_{n-1}\right\} \\
& \times \exp \left\{\log \left(\left(1-2 c_{3, n} \sigma_{n-1}^{2}\right)^{-1 / 2}\right)\right\} \exp \left\{\frac{c_{2, n}^{2} \sigma_{n-1}^{2}+4 \kappa_{n-1}^{2} \sigma_{n-1}^{2}+4 \mu_{n-1}^{2} S_{n-1}^{2} \sigma_{n-1}^{2}}{2\left(1-2 c_{3, n} \sigma_{n-1}^{2}\right)}\right\} \\
& \times \exp \left\{\frac{8 \kappa_{n-1} \mu_{n-1} S_{n-1} \sigma_{n-1}^{2}+4 c_{2, n} \sigma_{n-1}^{2} \kappa_{n-1}+4 c_{2, n} \sigma_{n-1}^{2} \mu_{n-1} S_{n-1}}{2\left(1-2 c_{3, n} \sigma_{n-1}^{2}\right)}\right\}, \\
= & \exp \left\{-a_{0, n-1}+c_{1, n}+c_{2, n} \kappa_{n-1}+c_{3, n} \kappa_{n-1}^{2}+\log \left(\left(1-2 c_{3, n} \sigma_{n-1}^{2}\right)^{-1 / 2}\right)\right. \\
& \left.+\frac{c_{2, n}^{2} \sigma_{n-1}^{2}+4 \kappa_{n-1}^{2} \sigma_{n-1}^{2}}{2\left(1-2 c_{3, n} \sigma_{n-1}^{2}\right)}+\frac{4 c_{2, n} \sigma_{n-1}^{2} \kappa_{n-1}}{2\left(1-2 c_{3, n} \sigma_{n-1}^{2}\right)}\right\} \\
& \exp \left\{S_{n-1}\left(-a_{1, n-1}+c_{2, n} \mu_{n-1}+c_{3, n} \kappa_{n-1} \mu_{n-1}+\frac{8 \kappa_{n-1} \mu_{n-1} \sigma_{n-1}^{2}+4 c_{2, n} \sigma_{n-1}^{2} \mu_{n-1}}{2\left(1-2 c_{3, n} \sigma_{n-1}^{2}\right)}\right)\right\} \\
& \exp \left\{S_{n-1}^{2}\left(-a_{2, n-1}+c_{3, n} \mu_{n-1}^{2}+\frac{4 \mu_{n-1}^{2} \sigma_{n-1}^{2}}{2\left(1-2 c_{3, n} \sigma_{n-1}^{2}\right)}\right)\right\} .
\end{aligned}
$$

Thus, by identification, we get

$$
\begin{aligned}
c_{1, n-1}:= & -a_{0, n-1}+c_{1, n}+c_{2, n} \kappa_{n-1}+c_{3, n} \kappa_{n-1}^{2}+\log \left(\left(1-2 c_{3, n} \sigma_{n-1}^{2}\right)^{-1 / 2}\right) \\
& +\frac{c_{2, n}^{2} \sigma_{n-1}^{2}+4 \kappa_{n-1}^{2} \sigma_{n-1}^{2}}{2\left(1-2 c_{3, n} \sigma_{n-1}^{2}\right)}+\frac{4 c_{2, n} \sigma_{n-1}^{2} \kappa_{n-1}}{2\left(1-2 c_{3, n} \sigma_{n-1}^{2}\right)}, \\
c_{2, n-1}:= & -a_{1, n-1}+c_{2, n} \mu_{n-1}+c_{3, n} \kappa_{n-1} \mu_{n-1}+\frac{8 \kappa_{n-1} \mu_{n-1} \sigma_{n-1}^{2}+4 c_{2, n} \sigma_{n-1}^{2} \mu_{n-1}}{2\left(1-2 c_{3, n} \sigma_{n-1}^{2}\right)}, \\
c_{3, n-1}:= & -a_{2, n-1}+c_{3, n} \mu_{n-1}^{2}+\frac{4 \mu_{n-1}^{2} \sigma_{n-1}^{2}}{2\left(1-2 c_{3, n} \sigma_{n-1}^{2}\right)} .
\end{aligned}
$$

which is the expected result.

Regarding (2.4), $\tilde{P}(k, T)$ is a function of the history of the Markov chain $X$ between time $k$ and $T-1$. Thus we can write $\tilde{P}\left(k, T, X_{k}, X_{k+1}, \ldots, X_{T-1}\right)$. Moreover, the coefficients $c_{1, k}, c_{2, k}$ and $c_{3, k}, k \in\{0,1, \ldots, T-1\}$ are measurable with respect to the $\sigma$-algebra generated by $X_{k}, X_{k+1}, \ldots$, and $X_{T-1}$. Consequently, they can be represented as functions of them. Hence 
we obtain for $k \in\{0,1,2, \ldots, T-1\}$

$$
\begin{aligned}
& c_{1, k}:=c_{1}\left(k, X_{k}\right)=c_{1}\left(k, X_{k}, X_{k+1}, \ldots, X_{T-1}\right), \\
& c_{2, k}:=c_{2}\left(k, X_{k}\right)=c_{2}\left(k, X_{k}, X_{k+1}, \ldots, X_{T-1}\right), \\
& c_{3, k}:=c_{3}\left(k, X_{k}\right)=c_{3}\left(k, X_{k}, X_{k+1}, \ldots, X_{T-1}\right) .
\end{aligned}
$$

This means by given $\tilde{\mathcal{G}}_{k}:=\mathcal{F}_{T}^{X} \vee \mathcal{F}_{k}^{S}$, the conditional bond price $\tilde{P}\left(k, T, X_{k}, X_{k+1}, \ldots, X_{T-1}\right)$ can be represented as follows:

$$
\begin{aligned}
& \tilde{P}\left(k, T, X_{k}, X_{k+1}, \ldots, X_{T-1}\right)= \\
& \exp \left\{c_{1}\left(k, X_{k}, X_{k+1}, \ldots, X_{T-1}\right)+c_{2}\left(k, X_{k}, X_{k+1}, \ldots, X_{T-1}\right) S_{k}+c_{3}\left(k, X_{k}, X_{k+1}, \ldots, X_{T-1}\right) S_{k}^{2}\right\} .
\end{aligned}
$$

Remark 2.2. In the specific case of an affine term structure of interest rate (i.e. $a_{2, k} \equiv 0$ in (1.2)), we have

$$
r_{k}:=r\left(k, X_{k}\right)=a_{0, k}+a_{1, k} S_{k}, \quad k \in \mathcal{T}
$$

And so the conditional bond price $\tilde{P}(k, T)$ admits also a affine structure form

$$
\tilde{P}(k, T)=\exp \left\{c_{1, k}+c_{2, k} S_{k}\right\}, \quad k \in \mathcal{T},
$$

where coefficient $c_{1, k}$ and $c_{2, k}$ satisfy the system of coupled stochastic backward recursions given for all $n \in\{1, \ldots, T-1\}$ by

$$
\begin{aligned}
c_{1, n-1} & :=-a_{0, n-1}+c_{1, n}+c_{2, n} \kappa_{n-1}+\frac{c_{2, n}^{2} \sigma_{n-1}^{2}}{2}+2 \kappa_{n-1}^{2} \sigma_{n-1}^{2}+2 c_{2, n} \sigma_{n-1}^{2} \kappa_{n-1}, \\
c_{2, n-1} & :=-a_{1, n-1}+c_{2, n} \mu_{n-1}+4 \kappa_{n-1} \mu_{n-1} \sigma_{n-1}^{2}+2 c_{2, n} \sigma_{n-1}^{2} \mu_{n-1} .
\end{aligned}
$$

with terminal conditions $c_{1, T}=c_{2, T}=0$ (see Duffie and Kan (1996) in [6] for more details about affine 
interest rate structure).

\subsection{General case}

In practice, we do not know the full history of the Markov chain $X$. Given the imperfect and/or incomplete information on all the future states of the economy. We need to evaluate our bond price given only the information set $\mathcal{G}_{k}$. Hence, following the representation (2.7) and the Theorem 2.1 we obtain the following result:

Proposition 2.1. Under the information set $\mathcal{G}_{k}$, the Bond price $P$ at time $k \in \mathcal{T}$ is given by

$$
P(k, T)=\sum_{i_{k}, i_{k+1}, \ldots, i_{T-1}=1}^{N}\left[\prod_{l=k}^{T-1} q_{i_{l} i_{l+1} k}\right] \tilde{P}\left(k, T, e_{i_{k}}, e_{i_{k+1}}, \ldots, e_{i_{T-1}}\right)
$$

where $\tilde{P}$ is given by (2.7) and coefficients $c_{i}\left(k, X_{k}, X_{k+1}, \ldots, X_{T-1}\right)$ for $i=\{1,2,3\}$ follow the recursive system given in Theorem 2.1

Proof. This result is obtained from taking the expectation of $\tilde{P}(k, T)$ conditioning on $\mathcal{G}_{k}$ and by enumerating all the transitions probabilities of the Markov chain $X$ from time $k$ to $T-1$.

\section{Conclusion}

We prove that if the short term interest rate follows a quadratic term structure of a regime switching asset process then the conditional zero coupon bond price with respect to the Markov switching process admits a quadratic term structure. Moreover, the stochastic coefficients appearing in this quadratic decomposition satisfy an explicit system of coupled stochastic backward recursions. This allows us to obtain an explicit way to evaluate this conditional zero coupon bond price. 


\section{References}

[1] Bansal, R. and Zhou, H. (2002). Term structure of interest rates with regime shifts. Journal of Finance, 57 (4), 1997-2043.

[2] Beaglehole D. and Tenney, M. (1991). General Solutions of Some Interest Rate- Contingent Claim Pricing Equations. Journal of Fixed Income 1, 69-83.

[3] Chen L., Filipovic, D. and Poor, H.V. (2004). Quadratic Term Structure Models for risk-free and defaultable rates. Mathematical Finance, 14(4), 515-536.

[4] Cox, Ingersoll, Ross, (1985). A Theory of the Term Structure of Interest Rates. Econometrica, $53,385-406$.

[5] Dai, Q. and Singleton, K. (2000). Specification Analysis of Affine Term Structure Mod-els. Journal of Finance, 55, 1943-1978.

[6] Duffie, D. and Kan, R. (1996). A yield-Factor Model of Interest Rates. Mathematical Finance, $6(4), 379-406$.

[7] Elliott, R.J., Aggoun, L. and Moore, J.B. (1994). Hidden Markov Models: estimation and control. Springer-Verlag, Berlin-Heidelberg- New York.

[8] Elliott, R.J, Siu, T.K. and Badescu, A. (2011). Bond valuation under a discrete-time regimeswitching term-structure model and its continuous-time extension. Managerial Finance, Vol. 37 Iss: $11,1025-1047$.

[9] Goutte, S. (2013). Pricing and hedging in stochastic volatility regime switching models. Journal of Mathematical Finance 3, 70-80.

[10] Goutte, S. (2014). Conditional Markov regime switching model applied to economic modelling. Economic Modelling, 38, 258-269. 
[11] Goutte, S. and Ngoupeyou, A. (2013). Defaultable Bond pricing using regime switching intensity model. Journal of Applied Mathematics and Informatics, 31 (3).

[12] Kirikos, D.G. (2000). Forecasting exchange rates out of sample: random walk vs Markov Switching Regimes. Applied Economics Letters 7, 133-136.

[13] Leblon, G. and Moraux, F. (2009). Quadratic Term Structure Models: Analysis and Performance. Paris December 2009 Finance International Meeting AFFI - EUROFIDAI.

[14] Leippold M. and Wu, L. (2002). Asset Pricing under the quadratic Class. Journal of Financial and Quantitative Analysis 37(2), 271-294.

[15] Li-Hsueh C. (2001). Inflation, Real Short-Term Interest Rates, and the Term Structure of Interest Rates: A Regime-Switching Approach. Applied Economics, 33, 393-400.

[16] Vasicek, O. (1977). An Equilibrium Characterization of the Term Structure. Journal of Financial Economics 5, 177-178. 